\title{
Bell's palsy: A case report, review and management
}

\author{
Narendra T Chaudhari \\ Dental Surgeon and Consulting Oral Pathologist at Private Practice, Mumbai, Maharashtra, India \\ *Corresponding Author: Narendra T Chaudhari \\ Email: naren.njoi@gmail.com
}

\begin{abstract}
Bell's palsy is defined as an idiopathic unilateral facial nerve paralysis, usually self-limiting. Bell's palsy was first described by Charles Bell and is the most common cause of acute facial nerve paralysis $(>80 \%)$. Immune, infective and ischemic mechanisms are all believed to play a role in the development of Bell's palsy, but the precise cause remains unclear. From corticosteroids to botulinum injections, wide therapeutic approaches have been employed for treating bell's palsy. Here, a case management and brief review of bell's palsy is discussed.
\end{abstract}

Keywords: Palsy, Idiopathic, Ischemic, Botulinum.

\section{Introduction}

Bell's palsy is the paralysis or severe weakness of the nerve that controls the facial muscles on the side of the face - the facial nerve or seventh cranial nerve. Patients typically find they suddenly cannot control their facial muscles, usually on one side. A relatively rare condition Bell's palsy has an annual incidence of 11 to 40 cases per 100000 population. It more commonly affects people over 15 and less than 60 years of age with both genders affected equally. ${ }^{1}$

Bell's palsy is an acute-onset peripheral facial neuropathy and is the most common cause of lower motor neuron facial palsy and cranial mononeuropathy. The clinical presentation of the disorder is a rapid onset, unilateral, lower motor neuron-type facial weakness with accompanying symptoms of postauricular pain, dysgeusia, subjective change in facial sensation and hyperacusis. Despite extensive study of the condition, the exact pathogenesis of Bell's palsy is still controversial. Infection (herpes simplex type-1), nerve compression and autoimmunity may all play a role, yet the exact sequence and magnitude of these influences remains unclear. ${ }^{2}$

The recurrence rate is $12 \%$, with a $36 \%$ recurrence rate on the same side. Risk factors include diabetes, hypertension, hypothyroidism, obesity and a compromised immune system. A family history is present in $4-8 \%$. Multiple recurrences are rare, and recurrence does not seem to be correlated with prognosis. Rarely, it can occur bilaterally. The prognosis is largely favourable. Management of Bell's palsy is controversial, because as many as two-thirds of patients recover spontaneously. Bell's palsy is normally treated using corticosteroids (prednisolone $1 \mathrm{mg} / \mathrm{kg}$ ) with or without antiviral agent (Acyclovir) and supplemented with vitamin B, physiotherapy and eye care. ${ }^{3}$

Treatment for Bell's palsy is etiology-driven if a specific case is identified. Most patients with Bell's palsy recover without treatment $71 \%$ achieve complete recovery, $84 \%$ achieve near normal function. However, $15 \%$ will be left with long-lasting sequelae, like poor recovery of facial muscle control and experience facial disfigurement, psychological trauma, and facial pain ${ }^{1}$ which can cause considerable psychological distress. ${ }^{4,5}$ A case report on bell's palsy along with brief review and management is discussed.

\section{Case Report}

A $25 y$ rs. old male patient reported to private dental clinic with the chief complaint of pain in lower right back tooth region along with swelling since a week that was treated by local physician with antibiotics and analgesics. Patient was referred to dental clinic by the physician for crooked smile.

Patient had history of prolonged exposure to cold wind. Patient had no history of vesicles in the ears and mouth. Patient was not a known diabetic or hypertensive and there was no other significant medical history and did not have any other systemic illnesses.

On extra-oral examination, patient shown deviation of lips towards left side while smiling, saliva drooling from right corner of mouth, patient was not able to hold air in mouth on right side and was not able to whistle, inability to close right eye completely, watering of the right eye, inability to raise the eyebrows on the right side. Fig. 1 (a-d). Patient had normal hearing and his speech was normal. Patient was advised OPG to rule out TMJ abnormalities and fracture.

Patient was suspected with Bell's palsy and referred to neurophysician for the confirmatory diagnosis. Patient was diagnosed as having bell's palsy (unilateral facial nerve paralysis) of the right side (lower motor neuron lesion) with the House-Brackmann facial nerve grading as Grade III moderate dysfunction and put under tapering dose of Predisolone along with multivitamins, eye patch, lubricating drops and was advised for physiotherapy and facial exercises. Patient completely recovered within 4 weeks. Fig. $2(\mathrm{a}-\mathrm{d})$ 

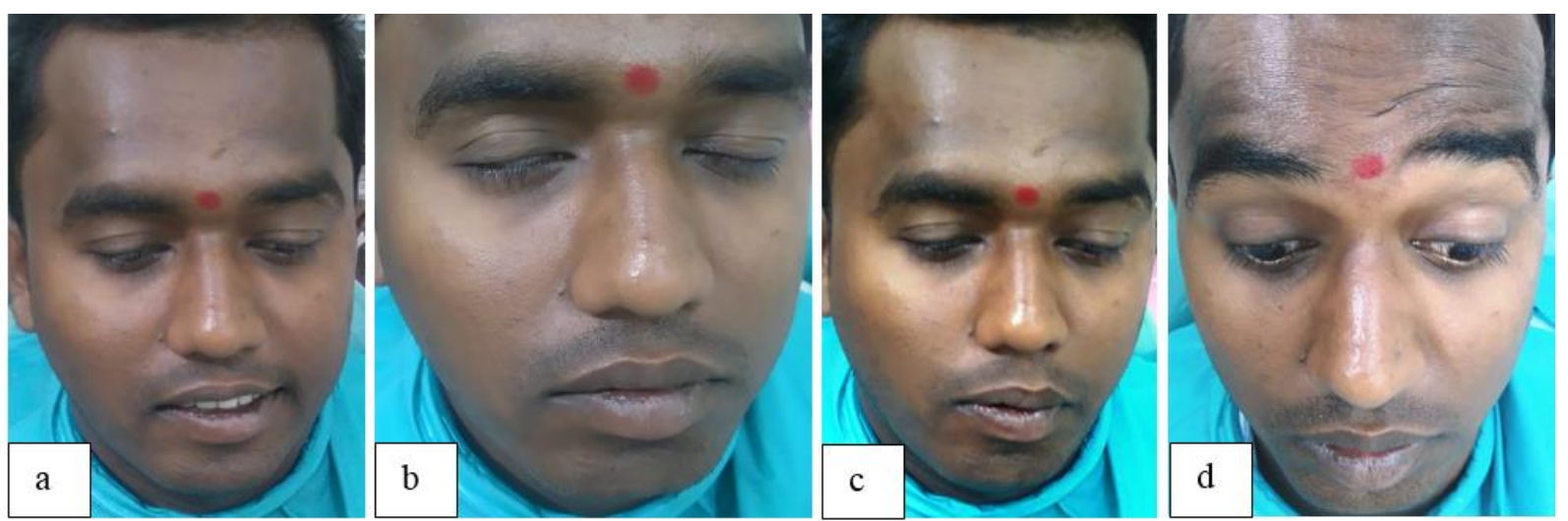

Fig. 1: Facial aspect of patient showing Bell's palsy on the right side; (a) Asymmetric smile; (b) Incomplete right eye closure; (c) Inability to hold air in mouth; (d) Absence of wrinkles in forehead and no movement of the eyebrow on right side
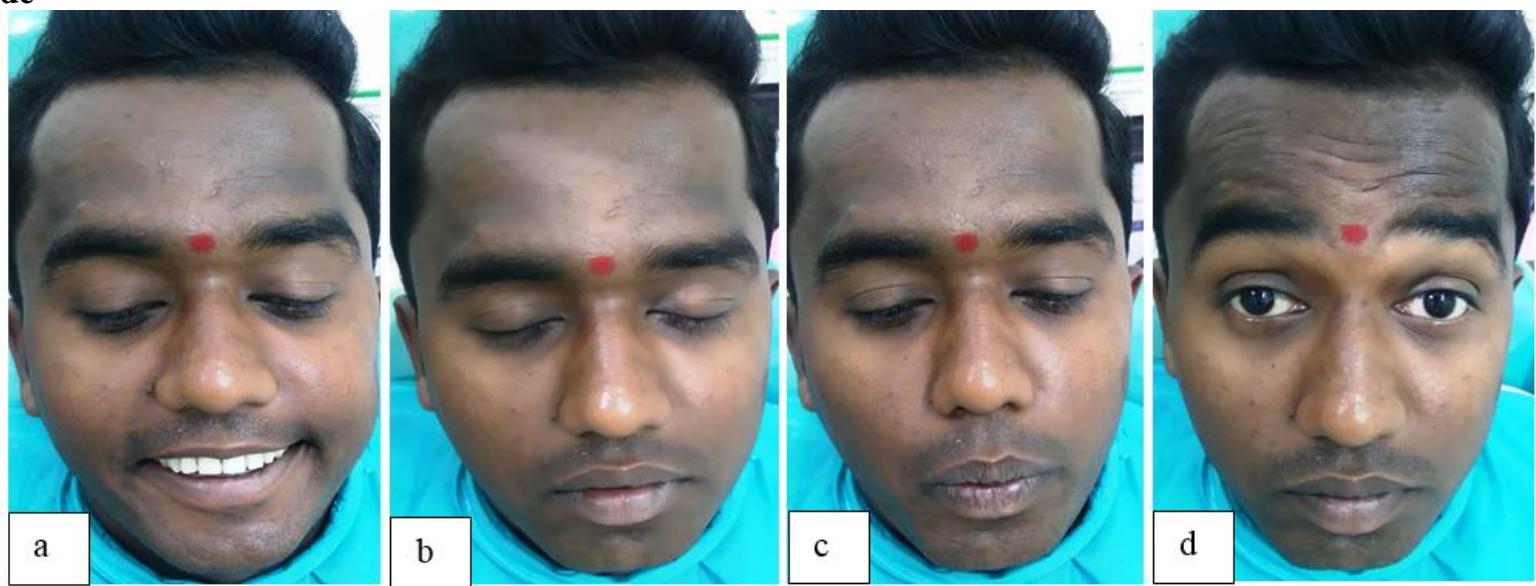

Fig. 2: Facial aspect of patient after recovery of facial movement; (a) Normal, symmetric smile; (b) Complete closure of the right eye; (c) Patient able to hold air in mouth; (d) Wrinkles in forehead and movement of the eyebrow on right side present

\section{Discussion}

Sir Charles Bell originally described this condition in 1821 as an acute-onset, idiopathic facial paralysis resulting from a dysfunction anywhere along the peripheral part of the facial nerve from the level of the pons distally. The exact cause of Bell's palsy is unknown but many theories are put forward like a virus similar to Herpes simplex or zoster, physiologic compression of the nerve due to arteriospasm, venous congestion or ischemia, and narrowing of the bony canal. Many case reports support a familial tendency suggesting the inheritance of an aberrant facial canal. ${ }^{6}$

The clinical features of Bell's palsy includes difficult or unable to close one of the eyelids, irritation in the eye because it does not blink and becomes too dry. Changes in the amount of tears the eye produces, drooping of corner of mouth on affected side, drooling from one side of the mouth. Decrease in salivary levels, difficulty with facial expressions, altered taste sensation, an affected ear may lead to sensitivity to sound, hyperacusis, pain in front or behind the ear on the affected side, headache. ${ }^{1}$

The most widely used systems for recording the severity of Bell's palsy are the House-Brackmann (HB) scale or the Facial Nerve Grading Scale (also known as the

Sunnybrook system). ${ }^{2}$ House-Brackmann has given different grading system in order to assess the degree of nerve damage which ranges from grade I to VI.

\section{Grade I - Normal facial function.}

Grade II- Mild dysfunction. Characteristics include Slight weakness is noted on close inspection, Slight synkinesis may be present, Normal symmetry and tone are noted at rest, Forehead motion is moderate to good, Complete eye closure is achieved with minimal effort, Slight mouth asymmetry is noted.

Grade III - Moderate dysfunction. The characteristics includes an obvious, but not disfiguring, difference is noted between the 2 sides, A noticeable, but not severe, synkinesis, contracture, or hemifacial spasm is present, Normal symmetry and tone are noted at rest, Forehead movement is slight to moderate, Complete eye closure is achieved with effort, A slightly weak mouth movement is noted with maximal effort.

Grade IV- Moderately severe dysfunction: Signs include an obvious weakness and/or disfiguring asymmetry is noted, Symmetry and tone are normal at rest, No forehead motion is observed, Eye closure is incomplete, an asymmetrical mouth is noted with maximal effort. 
Grade V- Severe dysfunction: Characteristics includes only a barely perceptible motion is noted, Asymmetry is noted at rest, No forehead motion is observed, Eye closure is incomplete, mouth movement is only slight.

Grade VI: Total paralysis with Gross asymmetry and No movement.

In this system, grades I and II are considered good prognosis, grades III and IV represent moderate dysfunction, and grades V and VI describe poor results. ${ }^{7}$

The differential diagnosis for facial palsy is broad, and misdiagnosis is common. Causes of facial palsy may be divided into congenital and acquired etiologies. Congenital causes include genetic syndromes, birth-related trauma and isolated disorders of development (e.g., developmental hypoplasia of facial muscles). Acquired causes include infective (VZV, Lyme disease, mycobacterium tuberculosis, HIV), traumatic (iatrogenic or head trauma), inflammatory (vasculitis, sarcoidosis, autoimmune disease), neoplastic (benign or malignant) and cerebrovascular causes. ${ }^{2}$ Diagnosis of Bell's palsy depends on clinical signs, symptoms and evaluation to exclude other possible causes of facial paralysis. Patients who have persistent clinical signs without improvement in facial paresis after 4 weeks, involvement of other cranial nerves or a second episode of palsy require further investigations. ${ }^{8}$ No specific laboratory test confirms the diagnosis of facial paralysis, its assessment remains clinical. A detailed history and thorough clinical examination should be carried out in those patients.

The aims of treatment in the acute phase of facial paralysis include strategies to speed recovery and to prevent corneal complications. Eye care includes eye patching and lubrication to protect the cornea from drying and abrasion secondary to poor lid closure and reduced tearing. Lubricating drops should be applied frequently during the day and an eye ointment should be used at night. Patients should be advised to use artificial tears to keep the eyes moist and prevent exposure keratitis. During the day, sunglasses are indicated, and dirty, noxious fumes should be avoided. During sleep, an ophthalmic ointment should be used. Strategies to speed recovery include physical therapy, corticosteroids and antiviral agents. ${ }^{9}$

The main goal of treatment is to improve the function of the facial nerve and reduce neuronal damage. In most of the cases, no treatment is required as it can spontaneously recover by itself. In the cases which are diagnosed at late stage or which requires treatment can be achieved with pharmacological, non- pharmacological or surgical means. Most of the patients can be treated with tapering dose of Prednisolone along with the antiviral drugs depending on the cause of onset of the bells palsy. According to the American Academy of Neurology [AAN] guidelines 2012, recommended dose of prednisone is $1 \mathrm{mg} / \mathrm{kg}$ or $60 \mathrm{mg} / \mathrm{day}$ for 6 days, followed by a taper, for a total of 10 days. But the steroids should be given cautionally in pregnancy, Diabetes mellitus, Renal or hepatic dysfunction and especially in the children's who are in the growth period. ${ }^{7}$

The most common treatment modality for Bell's palsy is corticosteroids, due to the fact that the mechanism of injury is inflammatory-mediated nerve damage. Recent studies shows significant treatment benefits in terms of both gain of complete recovery and reduction of long-term sequelae. Steroid treatment should be started within 72 hours of the onset of symptoms. Options include $25 \mathrm{mg}$ twice daily for ten days or $60 \mathrm{mg}$ daily for five days, followed by a daily reduction in dose of $10 \mathrm{mg}$ for a total treatment time of 10 days. Although a viral etiology is well documented in bell's palsy, there is currently no good evidence for antiviral agents (valaciclovir / acyclovir), either alone or in addition to a corticosteroid. It should be highlighted that although treatment with a corticosteroid plus an antiviral agent may lead to slightly higher recovery rates at three and six months compared to treating with a corticosteroid alone, the difference was not statistically significant. $^{3}$

Facial exercises by physiotherapist is recommended and performed while standing in front of a mirror and include trying to raise the eyebrows, opening and closing the eyes, blowing, and whistling, 5 times each, three times per day. The late phase of treatment is directed toward treating any residual facial movement deficit, and address in synkinesis, facial contractures, or autonomic dysfunction such as crocodile tears or hemi facial spasm. It is important for patients with Bell palsy to have a well-trained physiotherapist in the surrounding team to start training early after onset with correct activity. Facial exercises facilitate the return of intended facial movement patterns and eliminating unwanted patterns of facial movement and expression. ${ }^{10}$

\section{Conclusion}

Although its etiology is still unknown, viral infection, vascular ischemia and autoimmune disorders have all been postulated as possible mechanisms. Early recognition of signs and symptoms in consistent with Bell's palsy is important to avoid misdiagnosis. Differential diagnosis is essential to guide treatment in Bell's palsy. If the patient does not recover within the expected timeframe, imaging must be performed, such as computed tomography or magnetic resonance imaging to rule out cerebral pathologies. Special attention should be given to children with respect to steroid prescription.

Dentists, especially those who deal with children, should be aware of this disorder. The precise diagnosis of the cause of Bell's palsy and treating in its early stage helps in complete recovery of patient. The recurrence of Bell's palsy is rare in children and most of the patients recover in 3-4weeks.

\section{References}

1. Aishwarya Balakrishnan. "Bell's Palsy: Causes, Symptoms, Diagnosis and Treatment J" Pharm. Sci. \& Res. 2015;7(11):1004-1006.

2. Eviston TJ, Croxson GR, Kennedy PGE. "Bell's palsy: aetiology, clinical features and multidisciplinary care". $J$ Neurol Neurosurg Psychiatry. 2015;86:1356-136

3. Shawna Rekshmy D' dharan. "Facial Palsy - A Case Report." J Pharm Sci Res. 2016;8(10):1206-1209. 
4. Bianca Potterton. Bell's palsy: A Review" GP Registrar, Bridge Lane Surgery, London May/june 2015, 23-26.

5. McFarlin A, Peckler B. An unusual presentation of Bell's palsy: A case report and review of literature. J Emerg Trauma Shock. 2008;1:(1):50-52.

6. Keels MA, Long LM Jr., Vann WF Jr., Facial nerve paralysis: report of two cases of Bell's palsy. Am Acad Pediatr Dent. 1987;9(1):58-63.

7. Bali PK, Diavter V, Kalaivanan D, Puttaswamy V. Bell's Palsy: A Report of Two Cases. Int J Inf Res Rev. 2016;3(3):1965-1968.

8. Araujo MR, Azenha MR, Capelari, MM, Marzola C. Management of Bell's Palsy: A Report of 2 Cases. JADC. 2008;74(9):823-827.

9. AE Noor, N Amin, MTH Chowdhury, TM Shanta. An unusual presentation of Facial paralysis: A case report. Int J Appl Dent Sci. 2016;2(1)03-05.
10. Andrezza M CT, Carla M d S, Larissa A d S T, Adenilson d S daF, Fernando P L, Marco O, et al. Treatment of Bell Palsy using Facial Exercises in Primary Health Care: A Case Report. Biomed J Sci Tech Res. 2018; 3:4-. BJSTR.MS.ID.000944.

How to cite this article: Chaudhari N. T. Bell's palsy: A case report, review and management. J Oral Med, Oral Surg, Oral Pathol, Oral Radiol. 2018;4(4):190-193. 